\title{
EXACT DYNAMICAL SYSTEMS AND THE FROBENIUS-PERRON OPERATOR
}

BY

\author{
A. LASOTA AND JAMES A. YORKE ${ }^{1}$
}

\begin{abstract}
Conditions are investigated which guarantee exactness for measurable maps on measure spaces. The main application is to certain piecewise continuous maps $T$ on $[0,1]$ for which $T^{\prime}(0)>1$. We assume $[0,1]$ can be broken into intervals on which $T$ is continuous and convex and at the left end of these intervals $T=0$ and $d t / d x>0$. Such maps have an invariant absolutely continuous density which is exact.
\end{abstract}

1. Introduction. Exact dynamical systems describe irreversible deterministic processes with strong pseudo-stochastic behavior. It is natural, therefore, to study these processes from a statistical point of view as transformations of probability densities. This leads immediately to the idea of considering the properties of the corresponding Frobenius-Perron operators. We shall show that this idea is in fact fruitful and allows us to establish some simple, easily used, sufficient conditions for exactness.

The notion of exactness was introduced by V. A. Rochlin [8] who proved many interesting properties of exact dynamical systems, for example that any such system is mixing of all degrees and is a factor of a $K$ system. Rochlin has also presented some natural examples of exact systems. In particular he proved that the dynamical system corresponding to the $r$-adic transformation $(r>1)$

$$
T(x)=r x \quad(\bmod 1), \quad x \in[0,1],
$$

with the unique absolutely continuous (constructed by A. Rényi [7]) invariant measure is exact.

$\$ 2$ contains some preliminary notation. In particular we recall a necessary and sufficient condition for exactness of dynamical systems with a given invariant measure due to M. Lin [5]. This condition is stated in terms of the convergence of the iterates of the Frobenius-Perron operator. In $\S 3$ we weaken the assumption of convergence and show that using this operator it is actually possible, in some cases, to construct an absolutely continuous invariant measure such that the corresponding dynamical system is exact. This motivates the paper as we strive for a condition that is more easily applied. In the last section we give a construction of such a measure for piecewise convex mappings of the unit interval. This construction generalizes the results of Rényi and Rochlin concerning $r$-adic transformations.

Received by the editors October 27, 1980 and, in revised form, August 31, 1981.

1980 Mathematics Subject Classification. Primary 28D05.

${ }^{1}$ This research was supported in part under NSF grant MCS 781221A01.

(C)1982 American Mathematical Society 0002-9947/81/0000-0659/\$03.50 
2. Preliminaries. Let $(X, \Sigma, \mu)$ be a measure space with a normalized measure $\mu$ $(\mu(X)=1)$ and let $T: X \rightarrow X$ be a given transformation. In what follows we shall assume that $T$ is doubly measurable which means that $T(A) \in \Sigma$ and $T^{-1}(A) \in \Sigma$ for $A \in \Sigma$. In this section we shall also assume that $T$ is measure preserving, i.e. $\mu\left(T^{-1}(A)\right)=\mu(A)$ for $A \in \Sigma$. (One of the objectives of the next section is to eliminate this assumption.) An important role in our considerations is played by the $\sigma$-algebra

$$
\Sigma_{\infty}=\bigcap_{n=1}^{\infty} \Sigma_{n} \text { where } \Sigma_{n}=\left\{T^{-n}(A): A \in \Sigma\right\} .
$$

The dynamical system $(X, \Sigma, \mu ; T)$ is called exact if $\Sigma_{\infty}$ is trivial (it contains only the sets of measure zero and their compliments).

For a given $T$ we define the Frobenius-Perron operator $P$ corresponding to $T$ by

$$
\int_{A} P f d \mu=\int_{T^{-1}(A)} f d \mu \quad \text { for } A \in \Sigma \text { and } f=L^{1} \text {. }
$$

This formula has a simple probabilistic interpretation. Namely if $x$ is a random variable with the probability density function $f$, then $T(x)$ has probability density function $P f$. Observe that, according to the Radon-Nikodým Theorem, formula (1) defines the operator $P$ in a unique manner and may be used as a definition of $P$. In fact ( 1 ) is equivalent to

$$
P f=\frac{d}{d \mu} \nu \quad \text { where } \nu(A)=\int_{T^{-1}(A)} f d \mu
$$

and $d / d \mu$ denotes the Radon-Nikodym derivative. From (1) it can be seen that $P$ is linear and preserves the integral and is contractive in $L^{1}$ (that is, $\|P f\|_{L^{1}} \leqslant\|f\|_{L^{1}}$ ) even without the assumption that $\mu$ is invariant.

Exactness may be described in terms of the convergence of the iterates of the Frobenius-Perron operator. This fact was discovered by M. Lin who proved the following (M. Lin [5]) result. Let $\langle f, 1\rangle$ denote the integral of $f$.

THEOREM 1. Let $T: X \rightarrow X$ be a doubly measurable measure preserving transformation defined on a normalized measure space $(X, \Sigma, \mu)$. Then the following conditions are equivalent.

(a) The dynamical system $(X, \Sigma, \mu ; T)$ is exact;

(b) $\lim _{n} P^{n} f=\langle f, 1\rangle 1$ for each $f \in L^{1}(X, \Sigma, \mu)$ and the convergence is strong in $L^{1}$.

From the probabilistic interpretation of the operator $P$ it follows that the set

$$
D(X, \Sigma, \mu)=\left\{f \in L^{1}(X, \Sigma, \mu):\|f\|_{L^{1}}=1, f \geqslant 0\right\}
$$

plays a special role in the study of the properties of $P$. (The elements of $D$ will be called densities.) For example from Theorem 1 we immediately have

COROLlary 1. The system $(X, \Sigma, \mu ; T)$ is exact if and only if

(d) $\lim _{n} P^{n} f=1$ strongly in $L^{1}$ for every $f \in D(X, \Sigma, \mu)$. 
3. A sufficient condition for exactness. In this section we shall assume that $(X, \Sigma, \mu)$ is a $\sigma$-finite measure space and that $T: X \rightarrow X$ is doubly measurable and nonsingular. The last condition means that $\mu\left(T^{-1}(A)\right)=0$ whenever $\mu(A)=0$. For any density $f$ we shall denote by $\mu_{f}$ the measure

$$
\mu_{f}(A)=\int_{A} f d \mu \text { for } A \in \Sigma .
$$

Our goal now is to find conditions substantially weaker than (b) which still ensure the existence of a density $g$ for which the corresponding dynamical system $\left(X, \Sigma, \mu_{g} ; T\right)$ is exact. As in the previous section we define the operator $P$ : $L^{1}(X, \Sigma, \mu) \rightarrow L^{1}(X, \Sigma, \mu)$ by formula (1). Our starting point is the following (cf. [4]).

Proposition 1. Assume that a density $g$ has the property that

$$
\lim _{n} P^{n} f=g \text { for all } f \in D(X, \Sigma, \mu)
$$

and the limit is strong in $L^{1}(X, \Sigma, \mu)$. Then the measure $\mu_{g}$ is invariant and the system $(X, \Sigma, \mu ; T)$ is exact.

Proof. The function $g$ is evidently a fixed point of $P$ and from (1) it follows that $\mu_{g}$ is invariant. Now consider a new measure space $\left(X, \Sigma, \mu_{g}\right)$ and denote by $P_{0}$ the Frobenius-Perron operator corresponding to this space. We have

$$
\int_{A} P_{0}^{n} f d \mu_{g}=\int_{T^{-n}(A)} f d \mu_{g} \quad \text { or } \quad \int_{A}\left(P_{0}^{n} f\right) g d \mu=\int_{T^{-n}(A)} f g d \mu .
$$

This implies, according to the definition of $P$, the equality $g P_{0}^{n} f=P^{n}(f g)$. Now let $f \in D\left(X, \Sigma, \mu_{g}\right)$ be a bounded function. We have

$$
\int_{X}\left|P_{0}^{n} f-1\right| d \mu_{g}=\int_{X}\left|g P_{0}^{n} f-g\right| d \mu=\left\|P^{n}(f g)-g\right\|_{L^{1}}
$$

Since $f g \in D(X, \Sigma, \mu)$, the last term converges to zero. Thus the condition $P_{0}^{n} f \rightarrow 1$ is satisfied for all bounded functions $f$. By virtue of Corollary 1 this finishes the proof.

In the following theorem $(z)^{+}$denotes $\max \{0, z\}$.

Theorem 2. Let $(X, \Sigma, \mu)$ be a $\sigma$-finite measure space and let $T: X \rightarrow X$ be doubly measurable and nonsingular. Assume that there exists $h \in L^{1}(X, \Sigma, \mu), h \geqslant 0,\|h\|_{L^{1}}$ $>0$ such that

$$
\lim _{n}\left\|\left(h-P^{n} f\right)^{+}\right\|_{L^{1}}=0 \quad \text { for } f \in D(X, \Sigma, \mu) .
$$

Then there is a unique density $g$ such that the measure $\mu_{g}$ is invariant. Moreover condition (2) holds true and the system $\left(X, \Sigma, \mu_{g} ; T\right)$ is exact.

Before passing to the proof we shall introduce the following notion. A nonnegative function $h \in L^{1}(X, \Sigma, \mu)$ satisfying (3) will be called a lower function for $P$. Theorem 2 says that the existence of a nontrivial (different from zero) lower function for $P$ implies the existence of an invariant measure and the exactness of the 
corresponding dynamical system. Clearly $1 \geqslant\|h\|_{L^{1}}$ and in application it is easiest to find an $h$ of quite small norm. Condition (3) is weaker than (2) because the invariant density in (2) is an example of a lower function.

Proof of Theorem 2. By virtue of Proposition 1 it is sufficient to prove the existence of a function $g \in L^{1}(X, \Sigma, \mu)$ for which (2) holds. (The uniqueness of the invariant measure $\mu_{g}$ follows from (2) immediately.) This will be done in two steps. First we shall construct an increasing sequence $h \leqslant h_{1} \leqslant h_{2} \leqslant \cdots$ of lower functions converging to an invariant (under $P$ ) lower function $h^{*}$. Then we shall construct an increasing sequence $h^{*} \leqslant h_{1}^{*} \leqslant h_{2}^{*} \leqslant \cdots$ of invariant lower functions converging to an invariant density.

Step I. It is easy to see that the maximum of two arbitrary lower functions $\bar{h}$ and $\overline{\bar{h}}$ is a lower function. In fact setting $\tilde{h}=\max (\bar{h}, \overline{\bar{h}})$ we have

$$
\left\|\left(\tilde{h}-P^{n} f\right)^{+}\right\|_{L^{1}} \leqslant\left\|\left(\bar{h}-P^{n} f\right)^{+}\right\|_{L^{1}}+\left\|\left(\overline{\bar{h}}-P^{n} f\right)^{+}\right\|_{L^{1}}
$$

It is also obvious that for any lower function $\bar{h}$, the function $P \bar{h}$ is a lower function too. In fact from the equality

$$
P\left(h-P^{n-1} f\right)=P\left(h-P^{n-1} f\right)^{+}-P\left(P^{n-1} f-h\right)^{+}
$$

follows

$$
\left[P\left(h-P^{n-1} f\right)\right]^{+} \leqslant P\left(h-P^{n-1} f\right)^{+}
$$

and

$$
\left\|\left(P h-P^{n} f\right)^{+}\right\|_{L^{1}} \leqslant\left\|P\left(h-P^{n-1} f\right)^{+}\right\|_{L^{1}}=\left\|\left(h-P^{n-1} f\right)\right\|_{L^{1}} .
$$

Therefore, setting $h_{0}=h$ and $h_{n+1}=\max \left(h_{n}, P h_{n}\right)$ we define an increasing sequence of lower functions. Since $\left\|h_{n}\right\|_{L^{1}} \leqslant 1$, we have the strong (in $L^{1}$ ) limit

$$
h^{*}=\lim _{n} h_{n}
$$

exists. The function $h^{*}$ is also a lower function. In fact we have

$$
\left\|\left(h^{*}-P^{n} f\right)^{+}\right\|_{L^{1}} \leqslant\left\|h^{*}-h_{n}\right\|_{L^{1}}+\left\|\left(h_{m}-P^{n} f\right)^{+}\right\|_{L^{1}}
$$

The first term in the right-hand side is small for large $m$ (by the definition of $h^{*}$ ) and the second is small for large $n$ and fixed $m$ since $h_{m}$ is a lower function). It is also obvious from the inequality $P h_{n} \leqslant h_{n+1}$ that $P h^{*} \leqslant h^{*}$. Moreover we have $\left\|h^{*}\right\|_{L^{1}}=$ $\left\|P h^{*}\right\|_{L^{\prime}}\left(P\right.$ preserves the integral) and consequently $P h^{*}=h^{*}$.

Step II. We are going to show that if $h^{*}$ is an invariant lower function, then

$$
h_{1}^{*}=\left(2-\alpha_{0}\right) h^{*} \quad \text { where } \alpha_{0}=\left\|h^{*}\right\|_{L^{1}}
$$

has the same property. When $\alpha_{0}=1$, this fact is trivial. When $\alpha_{0}<1$, for a given $f \in D(X, \Sigma, \mu)$ consider the sequence

$$
q_{n}=\left(1-\alpha_{0}\right)^{-1} P^{n}\left(f-h^{*}\right)=\left(1-\alpha_{0}\right)^{-1}\left(P^{n} f-h^{*}\right) .
$$

Since $h^{*}$ is a lower function, we have

$$
\lim _{n}\left\|\left(-q_{n}\right)^{+}\right\|_{L^{1}}=0 \text { and } \lim _{n}\left\|q_{n}\right\|_{L^{1}}=1 .
$$


Therefore, for any given $\varepsilon>0$ there exists an integer $m>0$ and a function $r \in L^{1}(X, \Sigma, \mu)$ such that

$$
q_{m}+r \geqslant 0, \quad\left\|q_{m}+r\right\|_{L^{1}}=1, \quad\|r\|_{L^{1}} \leqslant \varepsilon / 2 .
$$

Since $q_{m}+r \in D(X, \Sigma, \mu)$ and $h^{*}$ is a lower function, we may conclude that

$$
\left\|\left(h^{*}-P^{n}\left(q_{m}+r\right)\right)^{+}\right\|_{L^{1}} \leqslant \varepsilon / 2
$$

for sufficiently large $n$, say $n \geqslant n_{0}$. Multiplication by $\left(1-\alpha_{0}\right)<1$ gives

$$
\left\|\left(\left(1-\alpha_{0}\right) h^{*}-P^{n+m} f-h^{*}+\left(1-\alpha_{0}\right) P^{n} r\right)^{+}\right\| \leqslant \varepsilon / 2 .
$$

Now from the inequality $\left\|P^{n} r\right\|_{L^{1}} \leqslant\|r\|_{L^{1}} \leqslant \varepsilon / 2$ it follows that

$$
\left\|\left(\left(2-\alpha_{0}\right) h^{*}-P^{n+m} f\right)^{+}\right\|_{L^{1}} \leqslant \varepsilon \text { for } n \geqslant n_{0}
$$

and finishes the proof that $h_{1}^{*}=\left(2-\alpha_{0}\right) h^{*}$ is a lower function. Of course

$$
\left\|h_{1}^{*}\right\|_{L^{1}}=\alpha_{0}\left(2-\alpha_{0}\right) \text {. }
$$

Let $\alpha_{1}=\alpha_{0}\left(2-\alpha_{0}\right)$. Repeating the above argument we may prove that $h_{2}^{*}=$ $\left(2-\alpha_{1}\right) h_{1}^{*}$ is also a lower function. This way we can construct a sequence of invariant lower functions

$$
h_{0}^{*}=h^{*}, \quad h_{n+1}^{*}=\left(2-\alpha_{n}\right) h_{n}^{*}
$$

where $\alpha_{0}=\left\|h^{*}\right\| \in(0,1)$ and $\alpha_{n+1}=\alpha_{n}\left(2-\alpha_{n}\right)$. An elementary calculation shows that $\lim _{n} \alpha_{n}=1$ and consequently

$$
\lim _{n}\left\|h_{n}^{*}\right\|_{L^{1}}=\lim _{n} \alpha_{n}=1 .
$$

The function $g=\lim _{n} h_{n}^{*}$ (the convergence is monotonic and strong in $L^{1}$ ) is again an invariant lower function and $\|g\|_{L^{1}}=1$. Consequently

$$
\left\|P^{n} f-g\right\|_{L^{1}}=\left\|\left(P^{n} f-g\right)^{+}\right\|_{L^{1}}+\left\|\left(g-P^{n} f\right)^{+}\right\|_{L^{1}}=2\left\|\left(g-P^{n} f\right)^{+}\right\|_{L^{1}} \rightarrow 0
$$

which finishes the proof.

It is interesting that the assumption on the existence of lower function in Theorem 2 may be replaced by an assumption of the existence of an upper function defined below. Thus we have the following

Theorem 3. Let $(X, \Sigma, \mu)$ be a $\sigma$-finite measure space and let $T: X \rightarrow X$ be doubly measurable and nonsingular. Assume that there exists $h \in L^{1}(X, \Sigma, \mu), h \geqslant 0,\|h\|_{L^{1}}$ $<2$ such that

$$
\lim _{n}\left\|\left(P^{n} f-h\right)^{+}\right\|_{L^{1}}=0 \quad \text { for } f \in D(X, \Sigma, \mu) .
$$

Then there is a unique density $g$ such that the measure $\mu_{g}$ is invariant. Moreover condition (2) holds true and the system $\left(X, \Sigma, \mu_{g} ; T\right)$ is exact.

Proof. By virtue of Theorem 2, it is enough to prove the existence of a lower function for $P$. To do this we again introduce an auxiliary notion. Every function $h \in L^{1}(X, \Sigma, \mu)$ for which condition (4) holds true will be called an upper function. As in the proof of Theorem 2, starting with a given upper function $h$ we define sequence

$$
h_{0}=h, \quad h_{1}=\inf \left(h_{0}, P h_{0}\right), \ldots, h_{n}=\inf \left(h_{n-1}, P h_{n-1}\right), \ldots
$$


of upper functions. The sequence $\left\{h_{n}\right\}$ is decreasing and bounded $\left(0 \leqslant h_{n} \leqslant h\right)$ and therefore convergent to a function $\tilde{h} \in L^{1}$. Once again it is easy to verify that $\tilde{h}$ is an upper function and that it is invariant, i.e. $P \tilde{h}=\tilde{h}$. Setting $\alpha=\|\tilde{h}\|_{L^{\prime}}$ we have $\alpha \leqslant\|h\|_{L^{1}}<2$. On the other hand from (4) it follows easily that $\alpha \geqslant 1$. Now we may consider two cases: (i) $\alpha=1$ and (ii) $1<\alpha<2$.

In case (i) the condition $\|\tilde{h}\|_{L^{1}}=1$ implies that

$$
\left\|\left(P^{n} f-h\right)^{+}\right\|_{L^{1}}=\left\|\left(h-P^{n} f\right)^{+}\right\|_{L^{1}} \quad \text { for } f \in D(X, \Sigma, \mu)
$$

and therefore, the function $\tilde{h}$ is simultaneously an upper function and a lower function. In this case the proof is finished.

In case (ii) we are going to show that $(2-\alpha) \tilde{h}$ is a lower function. For a given $f \in D(X, \Sigma, \mu)$ consider the sequence

$$
q_{n}=(\alpha-1)^{-1} P^{n}(\tilde{h}-f)=(\alpha-1)^{-1}\left(\tilde{h}-P^{n} f\right) .
$$

Since $\tilde{h}$ is an upper function we have

$$
\lim \left\|\left(-q_{n}\right)^{+}\right\|_{L^{1}}=0 \text { and } \lim _{n}\left\|q_{n}\right\|_{L^{1}}=1 .
$$

Thus for any given $\varepsilon>0$ there exists an integer $m>0$ and a function $r \in L^{1}(X, \Sigma, \mu)$ such that

$$
q_{m}+r \geqslant 0, \quad\left\|q_{m}+r\right\|_{L^{1}}=1, \quad\|r\|_{L^{1}} \leqslant \varepsilon / 2 .
$$

Again, since $q_{m}+r \in D(X, \Sigma, \mu)$ and $\tilde{h}$ is an upper function

$$
\left\|\left(P^{n}\left(q_{m}+r\right)-\tilde{h}\right)^{+}\right\|_{L^{1}} \leqslant \varepsilon / 2
$$

for sufficiently large $n$, say $n \geqslant n_{0}$. Multiplication by $\alpha-1<1$ gives, according to the definition of $q_{m}$,

$$
\left\|\left(\tilde{h}-(\alpha-1) \tilde{h}-P^{n+m} f+(\alpha-1) P^{n} r\right)^{+}\right\|_{L^{1}} \leqslant \varepsilon / 2 .
$$

Now from the inequality $\left\|P^{n} r\right\|_{L^{1}} \leqslant\|r\|_{L^{1}} \leqslant \varepsilon / 2$ it follows that

$$
\left\|\left((2-\alpha) \tilde{h}-P^{n+m} f\right)^{+}\right\|_{L^{1}} \leqslant \varepsilon \text { for } n \geqslant n_{0} .
$$

Thus $(2-\alpha) \tilde{h}$ is in fact a lower function.

RemarK 1. Since the operators $P^{n}$ are equicontinuous $\left(\left\|P^{n} f\right\|_{L^{1}} \leqslant\|f\|_{L^{1}}\right)$ for the validity of Theorems 2 and 3 it is enough to assume that the convergence in (3) and (4), respectively, holds only for all $f$ belonging to an arbitrary set $D_{0}$ dense in $D(X, \Sigma, \mu)$.

REMARK 2. The requirement that $\|h\|_{L^{1}}<2$ in Theorem 3 is the best possible in the sense that for $\|h\|_{L^{1}}=2$ the theorem is not valid. The simplest counterexample is given by the identity of mapping $T$ on the space $X=\left\{x_{0}, x_{1}\right\}$ with the measure $\mu$ defined by $\mu\left(\left\{x_{0}\right\}\right)=\mu\left(\left\{x_{1}\right\}\right)=\frac{1}{2}$.

REMARK 3. In the proofs of Theorems 2 and 3 the only properties of the operator $P$ which were used are:

(1) $P$ maps $L^{1}(X, \Sigma, \mu)$ into itself and is linear;

(2) $P f \geqslant 0$ for $f \geqslant 0, f \in L^{1}$;

(3) $\|P f\|_{L^{1}}=\|f\|_{L^{1}}$ for $f \geqslant 0, f \in L^{1}$. Therefore these theorems are valid for any 
operator $P$ satisfying (1), (2), and (3). (There exists a unique $g$ such that $P g=g$ and conditions (2) holds true.)

4. Piecewise convex transformations. The problem of the existence and uniqueness of an absolutely continuous measure $\mu_{(r)}$ for $r$-adic transformation

$$
T_{r}(x)=r x \quad(\bmod 1), \quad r>1,
$$

was solved by A. Rényi [7], A. O. Gelfond [2] and W. Parry [6]. In the special case, when $r$ is an integer, the ergodic properties of $T_{r}$ were studied by É. Borel [1]. The exactness of the dynamical system $\left([0,1], \mu_{(r)}, T_{r}\right)$ was proved by V. A. Rochlin [8]. These results were extended to some piecewise convex transformations in [3] and [4]. We shall show that using Theorem 2 it is possible to prove simultaneously the existence and uniqueness of absolutely continuous invariant measures and the exactness of corresponding dynamical systems for a fairly large class of piecewise convex transformations containing all previous cases. For a discussion of a special case of this result, see [6].

We say that a real valued function $F$ defined on an interval $\Delta$ is convex if

$$
F(\alpha x+(1-\alpha) y) \leqslant \alpha F(x)+(1-\alpha) F(y)
$$

for all $x, y \in \Delta$ and $0 \leqslant \alpha \leqslant 1$.

Let $T$ be a given transformation of the unit interval $[0,1]$ into itself. We shall assume that it satisfies the following conditions:

(i) there is a partition $0=a_{0}<\cdots<a_{N}=1$ such that for each integer $k$ $(k=1, \ldots, N)$ the restriction of $T$ to the interval $\left[a_{k-1}, a_{k}\right)$ is continuous and convex,

(ii) $T\left(a_{k-1}\right)=0, T^{\prime}\left(a_{k-1}\right)>0, k=1, \ldots, N$, and

(iii) $T^{\prime}(0)>1$.

TheOREM 4. Assume $T:[0,1] \rightarrow[0,1]$ satisfies conditions (i)-(iii). Then there exists the unique normalized absolutely continuous measure $\mu_{g}$ that is invariant under $T$. The system $\left([0,1], \mu_{g} ; T\right)$ is exact and the density $g=d \mu_{g} / d x$ is bounded and decreasing. Moreover

$$
\lim _{n} P^{n} f=g \quad \text { for } f \in D([0,1], \mu)
$$

where $\mu$ is the standard Borel measure on $[0,1]$ and $P$ is the Frobenius-Perron operator corresponding to $T$.

Proof. From formula (1) it follows that on the real line

$$
P f(x)=\frac{d}{d x} \int_{T^{-1}([0, x])} f(s) d s .
$$

For the transformation $T$ satisfying conditions (i)-(iii) the right-hand side of (4) can be easily calculated and we obtain

$$
P f(x)=\sum_{k=1}^{N} \psi_{k}^{\prime}(x) f\left(\psi_{k}(x)\right)
$$


where

$$
\psi_{k}(x)= \begin{cases}T_{k}^{-1}(x) & \text { for } x \in T\left(\left[a_{k-1}, a_{k}\right)\right), \\ a_{k} & \text { for } x \in[0,1] \backslash T\left(\left[a_{k-1}, a_{k}\right)\right)\end{cases}
$$

and $T_{k}$ denotes the restriction of $T$ to the interval $\left[a_{k-1}, a_{k}\right)$. The functions $\psi_{k}$ are increasing, continuous and differentiable except on a set of at most a countable number of points. At these points $\psi_{k}^{\prime}$ can be defined as the right-hand side derivatives. The functions $\psi_{k}^{\prime}$ are decreasing and bounded (since $T^{\prime}\left(a_{k}\right)>0$ ).

Now we are going to construct a nontrivial lower function for $P$. This will be done in three steps.

Step I. We start with the proof that the set

$$
S=\bigcup_{n=0}^{\infty} T^{-n}\left(\left\{a_{0}, \ldots, a_{N}\right\}\right)
$$

is dense in $[0,1]$. Suppose not. Then there exists an interval $\left[x_{0}, y_{0}\right] \subset(0,1)$ such that

$$
T^{n}\left(\left[x_{0}, y_{0}\right]\right) \cap\left\{a_{0}, \ldots a_{N}\right\} \text { is empty for all } n=0,1, \ldots .
$$

This means that for each $n$ the points $x_{n}=T^{n}\left(x_{0}\right)$ and $y_{n}=T^{n}\left(y_{0}\right)$ belong to the same interval $\left(a_{k-1}, a_{k}\right)$. If $x_{n}, y_{n} \in\left(a_{0}, a_{1}\right)$, we have by the convexity of $T_{1}$

$$
\frac{y_{n+1}}{x_{n+1}}=\frac{T_{1}\left(y_{n}\right)}{T_{1}\left(x_{n}\right)} \geqslant \frac{y_{n}}{x_{n}} \text {. }
$$

If $x_{n}, y_{n} \in\left(a_{k-1}, a_{k}\right)$ with $k>1$ we have a more precise inequality,

$$
\frac{y_{n+1}}{x_{n+1}}=\frac{T_{k}\left(y_{n}\right)}{T_{k}\left(x_{n}\right)} \geqslant \frac{y_{n}-a_{1}}{x_{n}-a_{1}} \geqslant \frac{y_{n}}{x_{n}}\left(\frac{1-a_{1} x_{n} / y_{n}}{1-a_{1}}\right)
$$

and consequently

$$
\frac{y_{n+1}}{x_{n+1}} \geqslant q \frac{y_{n}}{x_{n}} \quad \text { where } q=\frac{1-a_{1} x_{0} / y_{0}}{1-a_{1}}>1 .
$$

Since $T_{1}^{\prime}(x) \geqslant T_{1}^{\prime}(0)>1$, the points $x_{n}, y_{n}$ cannot belong to $\left(a_{0}, a_{1}\right)$ for almost all $n$. For infinitely many $n$ 's we have $x_{n}, y_{n}>a_{1}$ and, according to (7) and (8), $\lim _{n}\left(y_{n} / x_{n}\right)=\infty$. Since $\lim \sup _{n} x_{n} \geqslant a_{1}$, this in turn implies limsup $y_{n}=\infty$ which is impossible. The density of the set $S$ in $[0,1]$ is proved.

Step II. Let $1_{\Delta}$ be the characteristic function of an interval $\Delta=\left[d_{0}, d_{1}\right]$ with the endpoints belonging to the set $S$. We claim that for $n$ sufficiently large $P^{n} 1_{\Delta}$ is a decreasing function. Observe first that $P$ is the Frobenius-Perron operator corresponding to $T^{n}$. The function $T^{n}$ satisfies conditions analogous to (i)-(iii); in particular it is piecewise convex. Denote by

$$
0=a_{0}^{(n)}<\cdots<a_{N_{n}}^{(n)}=1
$$

the partition corresponding to $T^{n}$ (i.e. $T^{n}$ is convex on each interval $\left[a_{k-1}^{(n)}, a_{k}^{(n)}\right)$ and $\left.T^{n}\left(a_{k-1}^{(n)}\right)=0\right)$. It is easy to see that

$$
\left\{a_{0}^{(n)}, \ldots, a_{N_{n}}^{(n)}\right\}=T^{-n+1}\left\{a_{0}, \ldots, a_{N}\right\}
$$


if we assume for simplicity that $T(1)=0$. Moreover from the obvious inclusion

$$
\left\{a_{0}, \ldots, a_{N}\right\} \subset T^{-1}\left(\left\{a_{0}, \ldots, a_{N}\right\}\right)
$$

it follows by induction that

$$
T^{-n+1}\left(\left\{a_{0}, \ldots, a_{N}\right\}\right) \subset T^{-n}\left(\left\{a_{0}, \ldots, a_{N}\right\}\right)
$$

which shows that the system of partitions (9) is decreasing (finer for larger $n$ ). Since $d_{0}, d_{1} \in S$ there is an integer $n_{0}$ sufficiently large such that $d_{i}$ belongs to the partition $\left\{a_{0}^{(n)}, \ldots, a_{N_{n}}^{(n)}\right\}$ for $n \geqslant n_{0}$. The operator $P^{n}$ is the Frobenius-Perron operator for $T^{n}$ and so it may be written in the form analogous to (5), namely

$$
P^{n} f(x)=\sum_{k=1}^{N_{n}} \psi_{n, k}^{\prime}(x) f\left(\psi_{n, k}(x)\right)
$$

where $\psi_{n, k}\left(k=1, \ldots, N_{n}\right)$ denotes the inverse function to $T^{n}$ restricted to the interval $\left[a_{k-1}^{(n)}, a_{k}^{(n)}\right)$ (and $\psi_{n, k}$ is extended using a constant to the whole interval $[0,1]$ as in (6)). In particular for $f=1_{\Delta}$ and $n \geqslant n_{0}$ we have

$$
P^{n} 1_{\Delta}(x)=\sum_{k=k_{\alpha}}^{k_{\beta}-1} \psi_{n, k}^{\prime}(x)
$$

where $\alpha$ and $\beta$ are such that $a_{k_{\alpha}}^{(n)}=d_{0}$ and $a_{k_{\beta}}^{(n)}=d_{1}$. Since all the functions $\psi_{n, k}^{\prime}$ are decreasing, $P^{n} 1_{\Delta}$ has the same property.

Step III. Let $D_{0}$ be a subset of $D([0,1], \mu)$ consisting of all functions of the form

$$
f(x)=\sum_{k=1}^{n} c_{k} 1_{\Delta_{k}}(x), \quad c_{k} \geqslant 0
$$

where the endpoints of the intervals $\Delta_{k}$ belong to $S$. Since $S$ is dense in $[0,1]$, the set $D_{0}$ is dense in $D([0,1], \mu)$. Now we are in a position to construct a lower function for $P$. Let $f \in D_{0}$ be an arbitrary function. There exists $n_{0}=n_{0}(f)$ such that $P^{n} f$ is decreasing for $n \geqslant n_{0}$. It is easy to see that no decreasing density on $[0,1]$ exceeds $1 / x$. In fact for any decreasing $\bar{f}$ we have

$$
1 \geqslant \int_{0}^{x} \bar{f}(s) d s \geqslant \int_{0}^{x} \bar{f}(x) d s=x \bar{f}(x) .
$$

In particular we have $P^{n} f(x) \leqslant 1 / x$ for $n \geqslant n_{0}$. Applying this estimate to the equality

$$
P^{n+1} f(0)=\psi_{1}^{\prime}(0) P^{n} f(0)+\sum_{k=2}^{N} \psi_{k}^{\prime}(0) P^{n} f\left(a_{k-1}\right)
$$

we obtain

$$
P^{n+1} f(0) \leqslant \lambda_{1} P^{n} f(0)+\sum_{k=2}^{N} \frac{\lambda_{k}}{a_{k-1}}
$$

where $\lambda_{k}=\psi_{k}^{\prime}(0)=1 / T^{\prime}\left(a_{k-1}\right)$. From assumption (iii) we have $\lambda_{1}<1$ and by an induction argument we obtain

$$
P^{n+n_{0}} f(0) \leqslant\left(\lambda_{1}\right)^{n} P^{n_{0}} f(0)+\frac{M}{1-\lambda_{1}} \quad \text { where } M=\sum_{k=2}^{N} \frac{\lambda_{k}}{a_{k-1}} .
$$


Now let $K=M /\left(1-\lambda_{1}\right)+1$. For $n$ sufficiently large, say $n \geqslant n_{1}$, we have $P^{n} f(0)$ $\leqslant K$. Define $h=\frac{1}{2} 1_{[0,1 /(2 k)]}$. It is easy to prove that

$$
P^{n} f(x) \geqslant h(x) \text { for } n \geqslant n_{1} .
$$

Suppose not. Then there is $x_{0} \in[0,1 /(2 k)]$ such that $P^{n} f\left(x_{0}\right)<h\left(x_{0}\right)=\frac{1}{2}$ and consequently

$$
1=\int_{0}^{x_{0}} P^{n} f d x+\int_{x_{0}}^{1} P^{n} f d x<x_{0} k+\frac{1}{2}\left(1-x_{0}\right) \leqslant \frac{1}{2 K} K+\frac{1}{2}=1
$$

which is impossible. Inequality (10) finishes the proof.

\section{REFERENCES}

1. É. Borel, Les probabilités denombrables et leurs aplications aritmétiques, Rend. Circ. Mat. Palermo 27 (1909), 247-271.

2. A. O. Gelfond, A common property of number systems, Izv. Akad. Nauk SSSR Ser. Mat. 23 (1959), 809-814. (Russian)

3. A. Lasota, Invariant measures and functional equations, Aequationes Math. 9 (1973), 193-200.

4. __ A fixed point theorem and its application in ergodic theory, Tôhoku Math. J. 32 (1980), 567-575.

5. M. Lin, Mixing for Markov operators, Z. Wahrscheinlichkeitstheorie und Verw. Gebiete 16 (1971), 231-242.

6. W. Parry, On the $\beta$-expansion of real numbers, Acta Math. Acad. Sci. Hungar. 11 (1970), 401-416.

7. A. Rényi, Representation for real numbers and their ergodic properties, Acta Math. Acad. Sci. Hungar. 8 (1957), 477-493.

8. V. A. Rochlin, Exact endomorphisms of Lebesgue spaces, Izv. Akad. Nauk SSSR Ser. Mat. 25 (1971), 499-530; Amer. Math. Soc. Transl. (2) 39 (1964), 1-36.

Institute of Mathematics, Silesian University, 40-007 Katowice, Poland

Mathematics Department and Institute for Physical Science and Technology, University of Maryland, College Park, Maryland 20742 